\title{
Expert
}

\section{Commentary on the American College of Cardiology/ American Heart Association/Heart Rhythm Society 2008 Guidelines for Device-Based Therapy of Cardiac Rhythm Disorders}

\author{
A. Marc Gillinov, MD
}

From The Cleveland Clinic Foundation, Cleveland, Ohio.

Received for publication May 27, 2008; revisions received May 27, 2008; accepted for publication May 29, 2008.

Address for reprints: A. Marc Gillinov, MD, The Cleveland Clinic Foundation, Department of Thoracic/Cardiovascular Surgery, Desk F25, 9500 Euclid Avenue, Cleveland, OH 44195 (E-mail: gillinom@ccf.org).

J Thorac Cardiovasc Surg 2008;136:280-2 0022-5223/\$34.00

Copyright $(2008$ by The American Association for Thoracic Surgery

doi:10.1016/j.jtcvs.2008.05.007
$\mathrm{O}$ n May 15, 2008, The American College of Cardiology/American Heart Association/Heart Rhythm Society 2008 Guidelines for Device-Based Therapy of Cardiac Rhythm Abnormalities were published online. Subsequently, the guidelines were concurrently published in the Journal of the American College of Cardiology, Circulation, and Heart Rhythm. ${ }^{1}$ The guidelines were developed in collaboration with the American Association for Thoracic Surgery and the Society of Thoracic Surgeons. Focusing on 3 areas-pacemakers for bradyarrhythmias and heart failure management, cardiac resynchronization therapy (CRT), and implantable cardioverter defibrillators (ICDs) - these guidelines update the previous version published in 2002. The guidelines are comprehensive, and the reader is referred to appropriate sections for recommendations related to specific clinical presentations. This commentary will 1) provide a brief summary of several key recommendations, highlighting notable changes in the guidelines and their relevance to surgical practice, and 2) summarize the section on surgical considerations for device-based therapy.

\section{Key Recommendations \\ Pacemakers}

Indications for pacemaker therapy (eg, sinus node dysfunction and acquired atrioventricular block) are well defined. The guidelines encourage optimization of pacemaker programming to minimize unneeded right ventricular pacing. It is recognized that right ventricular apical pacing may not be the best approach in many patients because this mode of pacing has adverse effects on left ventricular (LV) and left atrial structure and function and is associated with an increased incidence of atrial fibrillation and congestive heart failure when compared with dual-chamber pacing. The role of biventricular pacing in patients without heart failure is not yet well defined, although this may be a reasonable option in selected young patients who require lifelong pacing. Pacemaker implantation is discouraged for asymptomatic bradycardia, particularly when bradycardia occurs at night. Pacing is not indicated for prevention of atrial fibrillation.

\section{Cardiac Resynchronization Therapy}

The need for optimization of medical therapy before CRT implantation is emphasized. CRT (with or without an ICD) is indicated (class IA recommendation) for patients who are in New York Heart Association (NYHA) class III or ambulatory class IV with optimal recommended medical therapy and who have a left ventricular ejection fraction (LVEF) less than or equal to $35 \%$, a QRS duration greater than or equal to 0.12 seconds, and sinus rhythm. Similar patients with atrial fibrillation may also 


\section{Abbreviations and Acronyms \\ CRT = cardiac resynchronization therapy \\ ICD = implantable cardioverter defibrillator \\ $\mathrm{LV}=$ left ventricular \\ LVEF $=$ left ventricular ejection fraction \\ NYHA $=$ New York Heart Association}

Surgically placed epicardial pacing leads are indicated in selected instances when standard transvenous lead placement is not feasible or contraindicated. Examples of such circumstances include 1) the inability or failure to place an adequate LV lead in patients requiring biventricular pacing, 2) indications for permanent pacing in certain pediatric patients and in pediatric or adult patients with tricuspid valve prostheses or recurrent or prolonged bacteremia, and 3) congenital or acquired venous anomalies that preclude transvenous access to the heart.

\section{Biventricular Pacing (Cardiac Resynchronization Therapy)}

The reported success rate of coronary venous lead implantation for CRT ranges from $81 \%$ to $99 \%$. Causes of failed percutaneous lead placement may be anatomic (superior vena cava or coronary sinus obstruction or inadequate coronary venous anatomy) or technical (failure to cannulate the coronary sinus, coronary sinus dissection, inadequately high pacing thresholds with intermittent capture, diaphragmatic pacing because of proximity of the phrenic nerve to the target coronary sinus branch, or lead dislodgement). When coronary sinus lead implantation fails, several nonrandomized studies have demonstrated that surgical LV lead placement is almost always successful. The key advantage of surgical lead placement is access to the entire posterior and lateral walls of the left ventricle, which enables choice of the best pacing site. The combination of echocardiography with tissue Doppler imaging and electrophysiologic measurements may facilitate the choice of a transthoracically directed LV epicardial pacing site. Placement of 2 epicardial leads may be considered to provide backup capability if one lead should fail or become dislodged. Steroid-eluting epicardial leads are generally preferable to screw-on leads.

The choice of surgical approach for LV epicardial leads seems to influence hospital morbidity. Surgical approaches for the placement of LV epicardial leads include left thoracotomy, left thoracoscopy, and robotically assisted portbased placement. Thoracotomy in frail patients with heart failure has been associated with bleeding, stroke, hypotension, and arrhythmias. In contrast, thoracoscopic and robotic approaches have been reported to be associated with minimal morbidity and are preferred. These less-invasive procedures generally require 60 to 90 minutes of operative time and a mean hospital stay of 4 to 5 days. However, not all patients are candidates for minimally invasive or robotic procedures. Patients who have undergone a previous thoracotomy or sternotomy may have limited pericardial/epicardial access.

In certain instances, it may be advisable to place an LV epicardial lead at the time of concomitant cardiac surgery. In patients who are currently or in the future may be candidates for CRT who require coronary artery bypass grafting or mitral valve surgery and who have medically refractory, symptomatic heart failure, ischemic or dilated 
cardiomyopathy, prolonged QRS interval, LV end-diastolic diameter greater than or equal to $55 \mathrm{~mm}$, and ejection fraction less than or equal to $35 \%$, the surgeon may elect to place an LV epicardial lead. The lead is tunneled to a prepectoral pocket for intraoperative or postoperative attachment to an appropriate pacing generator. This approach is probably not indicated for the patient who is expected to have substantial improvement in LVEF after cardiac surgery (eg, a patient with extensive viable myocardium undergoing revascularization). There are limited data documenting outcomes of this "preemptive" strategy.

\section{Pediatric Patients}

Epicardial leads may be necessary in some pediatric patients. The most common indications for permanent pacemaker implantation in the pediatric population are sinus node dysfunction or atrioventricular block after surgery for congenital heart disease or congenital atrioventricular block. In most instances, such pacing systems can be placed using standard transvenous techniques. However, epicardial leads may be needed in children as a result of their small size, the presence of congenital heart defects with a right-to-left shunt, or an inability to pace the chamber desired because of anatomic barriers (eg, after a Fontan procedure). In such instances, steroid-eluting leads provide excellent durability.

\section{Bacteremia}

Epicardial leads are suggested in some pediatric or adult patients who need pacing and who have recurrent or prolonged bacteremia. For a single episode of device-related bacteremia, extraction of all hardware followed by reimplantation by the transvenous route at a later date is appropriate.

\section{Tricuspid Valve Surgery}

Implantation of permanent epicardial pacing leads is indicated in the pacemaker-dependent patient undergoing tricuspid valve replacement. A mechanical tricuspid valve represents an absolute contraindication to placement of transvenous right ventricular leads, because such leads will cross the valve and interfere with valve function. This scenario occurs most commonly in the patient with tricuspid valve endocarditis and a transvenous pacemaker. At surgery, all hardware should be removed. If the tricuspid valve is reparable, standard transvenous pacing leads can be placed postoperatively. However, if tricuspid valve replacement is necessary, epicardial ventricular leads should be implanted at the time of surgery.

\section{Conclusions}

With appropriate training, surgeons can place both endovascular and epicardial leads and corresponding generators. The newly issued American College of Cardiology/American Heart Association/Heart Rhythm Society 2008 Guidelines for Device-Based Therapy of Cardiac Rhythm Abnormalities ${ }^{1}$ provide detailed, well-referenced recommendations to guide patient selection and device type.

\section{Reference}

1. Epstein AE, DiMarco JP, Ellenbogen KA, et al. ACC/AHA/HRS 2008 Guidelines for Device-Based Therapy of Cardiac Rhythm Abnormalities: a report of the American College of Cardiology/American Heart Association Task Force on Practice Guidelines (Writing Committee to Revise the ACC/AHA/NASPE 2002 Guideline Update for Implantation of Cardiac Pacemakers and Antiarrhythmia Devices). J Am Coll Cardiol. 2008; 51:e1-62. 